\title{
Iberian electricity market ontology to enable smart grid market simulation
}

\author{
Gabriel Santos ${ }^{1}$, Tiago Pinto ${ }^{2 *}$, Isabel Praça ${ }^{1}$ and Zita Vale ${ }^{3}$
}

\author{
*Correspondence: tpinto@usal.es \\ ${ }^{2}$ BISITE research group - University \\ of Salamanca, Calle Espejo, s/n, \\ 37007 Salamanca, España \\ Full list of author information is \\ available at the end of the article
}

\begin{abstract}
Several approaches have been proposed to enhance the potential of distributed generation (DG). Some of the most prominent solutions include the aggregation of DG units and other players, culminating in the concept of and Smart Grid (SG). In this context, several simulation tools arose to study and test the new market mechanisms. However, all of these simulators are closed and centred in their object of study, neglecting the potential advantages of interoperating with other systems from the same domain. This work proposes the use of ontologies for systems interoperability in the power and energy systems domain. The ontologies have been developed and implemented in MASCEM and MASGriP - multi-agent simulators of electricity markets, and SG operation and management,respectively; thus enabling joint electricity market and SG simulations.
\end{abstract}

Keywords: Iberian electricity market, Multi-agent simulation, Ontology, Semantic interoperability

\section{Introduction}

The deregulation of electricity markets (EM), the high penetration of distributed generation (DG) units as well as other distributed energy resources, require more intelligent operation methods. This brings out several important issues of both economic and technical nature that need to be addressed (Kakran and Chanana 2018). The coordination of a system of this type is quite challenging, requiring distributed intelligence. Dealing with such issues has led to the birth of the concept of Smart Grids (SG) (Vale et al. 2011).

To study and analyse these types of operations, multi-agent systems (MAS) are often used as simulation tools. For this purpose we use the Multi-Agent Smart Grid simulation Platform (Oliveira et al. 2012; Gomes et al. 2014) (MASGriP), a multi-agent tool that models the operation of SG. To efficiently analyse the quality of the simulated SG management, it is essential to provide the means for MASGriP to be able to participate in EM negotiations. To this end, we use the Multi-Agent Simulator of Competitive Electricity Markets (Praça et al. 2003; Santos et al. 2016a) (MASCEM), developed for studying, analysing and testing the operation of the complex and restructured EM. The interoperability between MASGriP and MASCEM is assured by the use of semantics (Veeckman et al. 2017; Ritschel et al. 2016; Bennaceur et al. 2015). More specifically by using MASCEM's ontologies, which are publicly available ${ }^{1}$ (Santos et al. 2016a, b, c).

(c) The Author(s). 2018 Open Access This article is distributed under the terms of the Creative Commons Attribution 4.0 International License (http://creativecommons.org/licenses/by/4.0/), which permits unrestricted use, distribution, and reproduction in any medium, provided you give appropriate credit to the original author(s) and the source, provide a link to the Creative Commons license, and indicate if changes were made. 
This work disseminates the use of ontologies for the interoperability of heterogeneous agent based tools in the scope of the wholesale EM, while introducing the MIBEL Ontology - a module extended from the Electricity Markets Ontology (Santos et al. 2016a, c) to enable MASCEM interoperability in the simulation of the Iberian EM - MIBEL. This work results from the findings of the work presented in (Santos 2015).

The following section overviews the most relevant related work. "MIBEL ontology" section introduces the MIBEL Ontology. "Case study" section presents a case study based on real data to prove the usefulness of using semantics for multi-agent interoperability. Finally, "Conclusions" section features the final conclusions.

\section{Related work}

EM are evolving to regional markets and some to continental scale. Nowadays, a considerable amount of electrical energy is enabled by the efficient use of renewable based generation in places where it exceeds the local needs (Sioshansi 2013).

The shared interest of regulators and market players in foreseeing and analysing the market's behaviour requires a clear understanding of EM principles, and the impact of power systems physics on market dynamics and vice-versa (Meeus et al. 2005; Biggar and Hesamzadeh 2014). For the success of all involved players, a suitable understanding of the diversity of market types and regulatory models that have been introduced is critical. In this scope arise several modelling and simulation tools, such as MASCEM and MASGriP.

MASCEM (Praça et al. 2003; Santos et al. 2016a) is simulation and modelling tool developed to study and simulate EM operation. MASCEM models the main market entities, such as: market and system operators, buyer and seller agents (consumers, producers and/or prosumers), and aggregators; and their interactions. Medium/long-term gathering of data and experience is also considered to support players' decisions according to their specific characteristics and goals. The main market entities are implemented as software agents.

The main types of negotiations normally present in EM included in MASCEM are: dayahead and intraday pool (symmetric or asymmetric, with or without complex conditions) markets, bilateral contracts and forward markets. By selecting a combination of these market models, it is also possible to perform hybrid simulations. MASCEM allows the simulation of three of the main European EM: MIBEL ${ }^{2}$, EPEX $^{3}$ and Nord Pool ${ }^{4}$.

MASGriP (Oliveira et al. 2012; Gomes et al. 2014) is a MAS that models the internal operation of SG and Microgrids (MG). The involved players are represented by software agents. It allows testing and analysing different types of models, namely energy resource management methodologies, contract negotiation methods, energy transaction models, and diverse types of Demand Response (DR) programs and events.

Most players in MASGriP represent energy resources such as: different types of consumers and producers; Electric Vehicles with vehicle-to-grid capabilities, among others. The Distribution System Operator (DSO) and the Independent System Operator (ISO) have also been included to reflect the real world. Its integration with MASCEM allows the simulation platform to go a step further, including the EM simulation capabilities for joint simulations.

The Iberian Electricity Market (MIBEL) is one of the most liquid markets in Europe (OMIE 2017). It is treated as a single system defining the same market price for both Portugal and Spain. However, when congestions occur a splitting mechanism is used, which 
may result in a distinct market price per area. A bid can be carried out based on 25 offers per period. Regular bids feature for each offer a price and amount of power (OMIE 2012).

In MIBEL, the complex conditions are different from other important EM and depend on the type of market to execute (OMIE 2017; Santos et al. 2011, 2012). In the case of the day-ahead market, only sellers may present complex conditions, while in the intraday market both buyers and sellers may present them.

Regarding the intraday market, although being very similar to the daily market, it contains 6 market sessions, where players can renegotiate previously negotiated periods in the spot market, in order to fit their needs. In this market type buyers are allowed to sell and sellers are allowed to buy (OMIE 2017). In intraday markets different complex conditions are available for both buyers and sellers. More details on MIBEL's day-ahead and intraday complex conditions are available in (Santos et al. 2011, 2012).

It is possible to find in literature a few solutions able to promote interoperability among heterogeneous systems, in the power and energy systems area, such as the Electric Power and Communication Synchronizing Simulator (EPOCHS) (Hopkinson et al. 2006), the Global Event-Driven Co-Simulation framework (GECO) (Lin et al. 2012), and Mosaik system (Schütte et al. 2011; Scherfke 2018). EPOCHS (Hopkinson et al. 2006) is an agent-based system that uses realistic scenarios to combine the results of several simulators, solving network communication problems and avoiding potential costs. GECO (Lin et al. 2012) models and simulates the monitoring, protection and control schemes of the power systems and respective communication network. Mosaik (Schütte et al. 2011; Scherfke 2018) provides models to simulate producers, consumers, prosumers, electric vehicles and other elements of the network, enabling the simulation of heterogeneous SG components. It addresses many of the challenges of interoperability but is only able to perform static simulations previously configured and programmed. To add a new system to the simulation, it must be reprogrammed accordingly.

Interoperability between systems is highly complex due to their heterogeneity. It is necessary to establish a communication mechanism enabling the information sharing, while given the same meaning to common concepts, their properties and attributes. Ontologies provide a common understanding on a given domain, by modelling their concepts, properties and relations (Man 2013). Several ontologies have been proposed in the power and energy systems field (Schachinger et al. 2016; Hippolyte et al. 2016; Maffei et al. 2017; Alexopoulos et al. 2009). However, in the EM domain, there was only one work developed for the greek EM and adapted to the EU directives (Alexopoulos et al. 2009). It is mainly focused on the EM's legislation. Besides not being publicly available for extension or reuse, it also is out of the scope of multi-agent EM simulation. For this reason, the authors developed from scratch the semantic model that is the basis for this work (Santos et al. 2016a). This work extends previous work by defining a specific semantic model for the Iberian EM.

\section{MIBEL ontology}

The MIBEL Ontology (MBL) ${ }^{5}$ was the first extension of EMO to be developed. The Iberian market has been the first EM to be implemented in MASCEM and it is under continuous development ever since. It is therefore important to keep this ontology as flexible as possible in order to meet its constantly evolving nature. 
The MBL ontology imports EMO, extending some of EMO's concepts - namely Bid, Market, MarketType and Session - and including the ComplexConditions class and subclasses. Figure 1 illustrates the classes extended and defined in MBL, highlighted with red rounded rectangles.

As it is possible to observe by Fig. 1, the concepts BuyBid, InvalidRegularBid, RegularBid and SaleBid are extended from EMO:Bid. From EMO:Market arises the MIBEL concept and from EMO:MarketType the Day-Ahead and Intraday subclasses. The EMO:Session is super class of DayAheadSession and IntradaySession. The ComplexConditions object is included as a new concept together with its particular subclasses, namely: CompleteAcceptanceInEachHourInTheMatchingPeriodOfTheFirstBlockOfTheBid, CompleteAcceptanceInTheMatchingProcessOfTheFirstBlockOfTheBid, Indivisibility, LoadGradient, MaximumMatchedPower, MaximumPayment, MinimumIncome, MinimumNumberOfConsecutiveHoursOfCompleteAcceptance OfTheFirstBlockOfTheBid and ScheduledStop, representing the different types of complex conditions available in the Iberian market.

MBL also includes new object and data properties with respect to the complex conditions. Figure 2 features the new object and data properties included in MBL.

On the left part of Fig. 2, the new object properties are highlighted, namely the hasComplexCondition, incorporatedByPlayer and incorporatesComplexCondition; and on the right part the new data property valuePerMWh is highlighted. All these new properties are related to the new concept of ComplexConditions and will be further explained hereinafter.

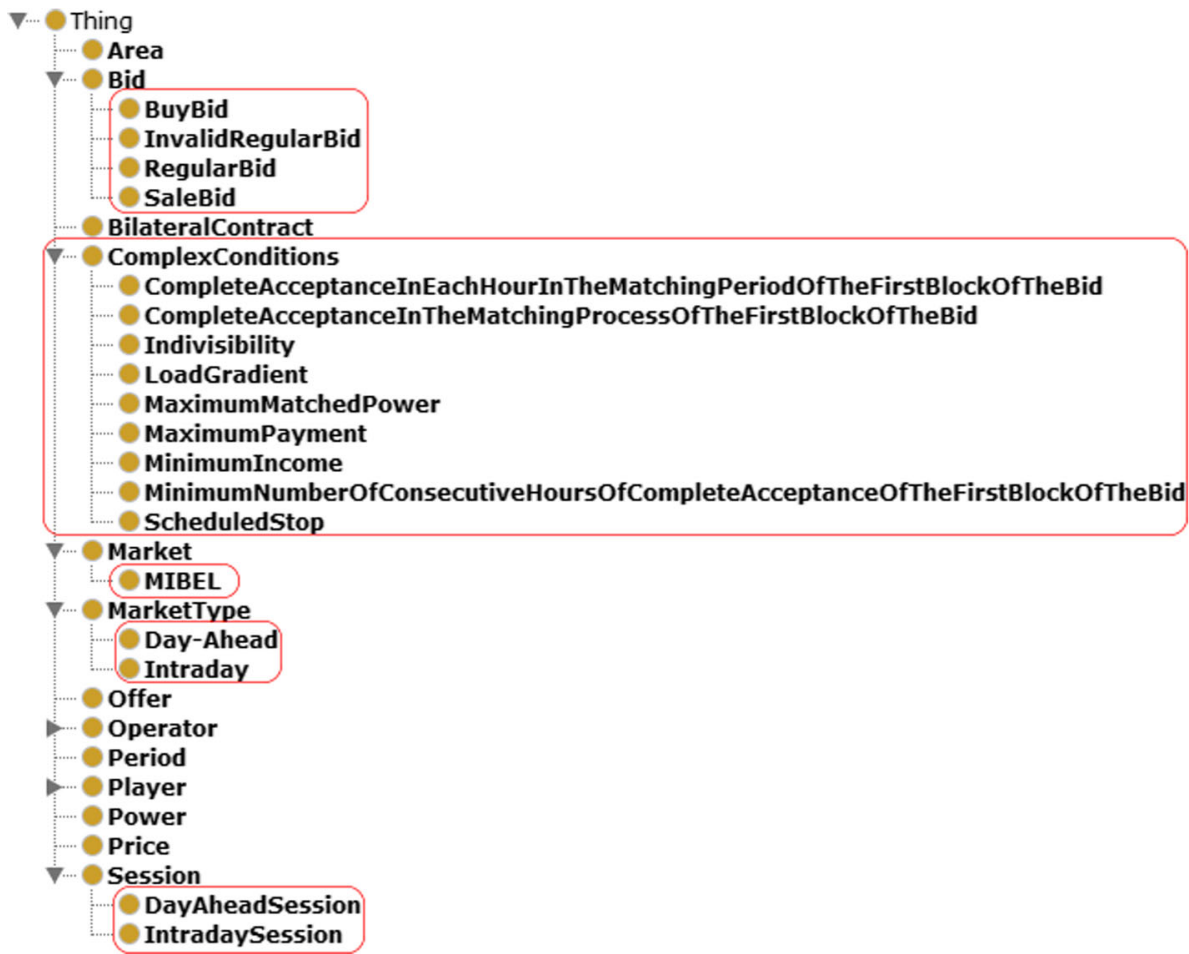

Fig. 1 MIBEL Ontology classes 


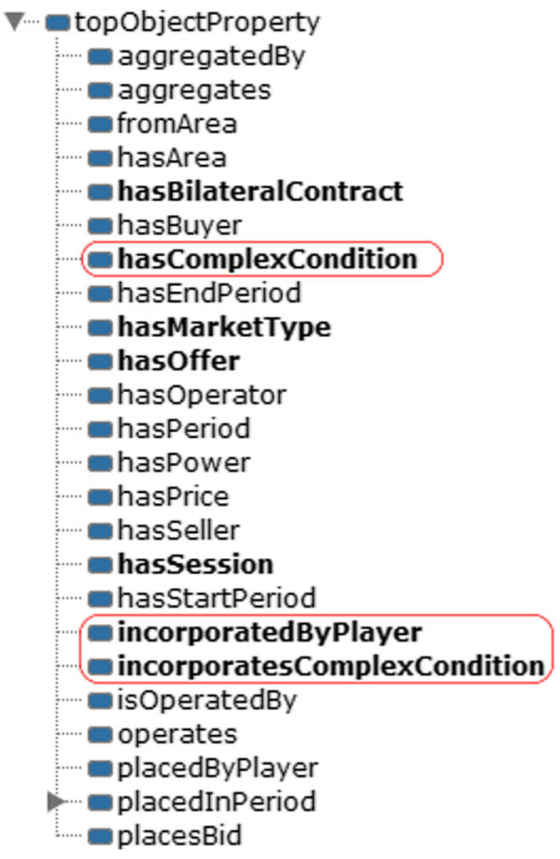

Fig. 2 MIBEL Ontology object and data properties

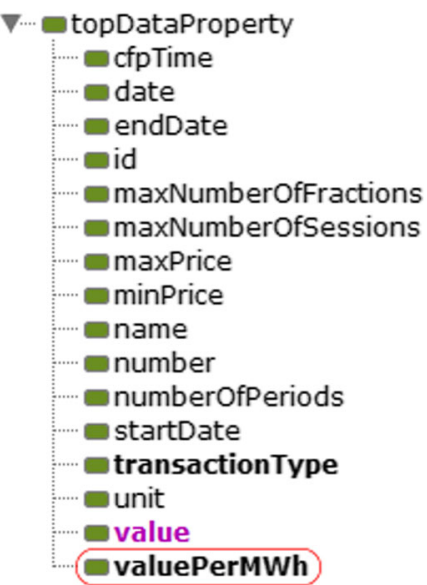

The MBL ontology is illustrated in Fig. $3^{6}$ where the classes, object properties and data property identified above are represented. The existing relations between them and between them and the EMO's concepts and properties are also shown.

The yellow rectangles represent the concepts imported from EMO. In blue are the object properties. If imported from EMO they use the prefix "EMO:". The inferred object property incorporatesComplexCondition, which is an inverse property of incorporatedByPlayer is represented in orange. The relations between the remaining EMO concepts have been left out in order to simplify the reading of the diagrams, since they have already been presented in Santos et al. (2016a, b, c).

From Fig. 3 it is possible to observe that the different complex conditions are distributed between the distinct market types. Both market types have different constraints in MIBEL, depending on if it is a day-ahead or intraday session. On the other hand, MIBEL only makes available one type of Bid, the RegularBid which can only be placed in one EMO:Period (placedInSinglePeriod data property) and contain a maximum of 25 EMO:Offers. BuyBid and SaleBid are types of bids inferred depending on their transactionType data property, while an InvalidRegularBid represents a Bid with more than 25 fractions (EMO:Offer).

This ontology is used both by players and by the market operator. The market operator detains a knowledge base defining the market's features. This knowledge base is created from the user's input file. The same input file also determines the players' knowledge bases. The market operator gathers the players' proposals using the ontology's conceptualization.

The MBL ontology expressiveness is the same of EMO: $A L C H I Q(D)$ (Santos et al. 2016a, c). Tables 1, 2 and 3 present the object properties, data property and classes 


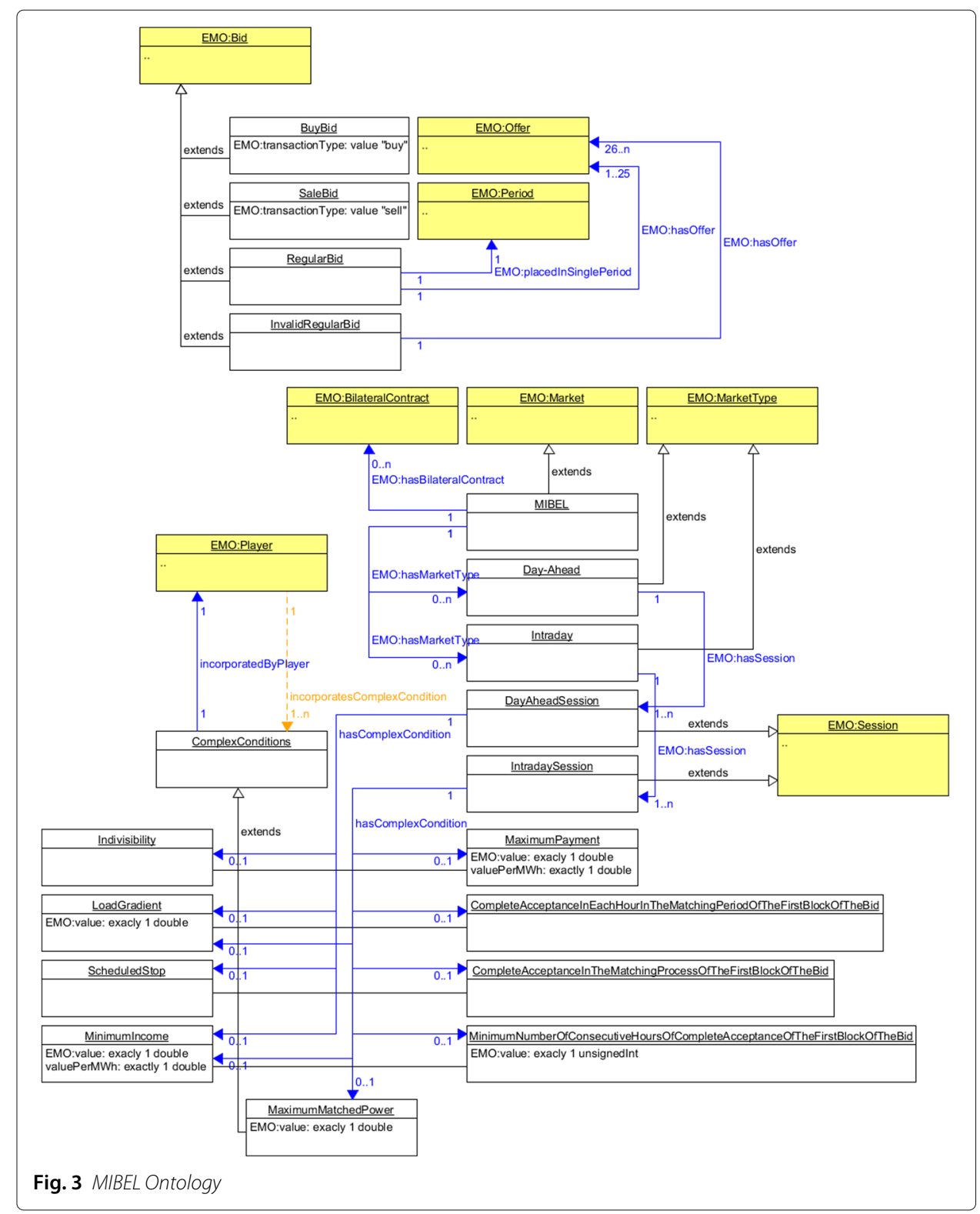

of the MBL ontology in description logics (DL) syntax ${ }^{7}$, respectively. DL is a common knowledge representation language which enables formalizing and structuring semantic models in a well-understood way (Dong et al. 2017; Baader et al. 2008).

The hasComplexCondition property is sub-property of $R . R$ in DL syntax represents the top level abstract object property. The incorporatedByPlayer property is also

Table 1 MIBEL Ontology object properties DL syntax

\begin{tabular}{l} 
Object Properties \\
\hline hasComplexCondition $\subseteq R$ \\
incorporatedByPlayer $\sqsubseteq R$ \\
$T \sqsubseteq \leq 1$ incorporatedByPlayer \\
incorporatesComplexCondition $\equiv$ incorporatedByPlayer $^{-}$ \\
$T \subseteq \leq 1$ incorporatesComplexCondition \\
\hline
\end{tabular}


Table 2 MIBEL Ontology data property DL syntax

\begin{tabular}{l} 
Data Property \\
valuePerMWh $\subseteq \cup$ \\
$\top \sqsubseteq \leq 1$ valuePerMWh \\
\hline
\end{tabular}

sub-property of $R$, and it is a Functional ${ }^{8}$ object property. The incorporatesComplexCondition object property is symmetric to the incorporatedByPlayer property, being also Functional.

The only data property added to EMO is the valuePerMWh property. In addition to be sub-property of $\cup$, it is also a Functional property. $\cup$ in DL is the top level abstract data property.

BuyBid and SaleBid are subclasses of EMO:Bid being defined by the transactionType data property, which is equal to "buy" or "sell" respectively. A RegularBid is also a subclass of EMO:Bid but including a maximum number of 25 EMO:Offers using

Table 3 MIBEL Ontology classes DL syntax

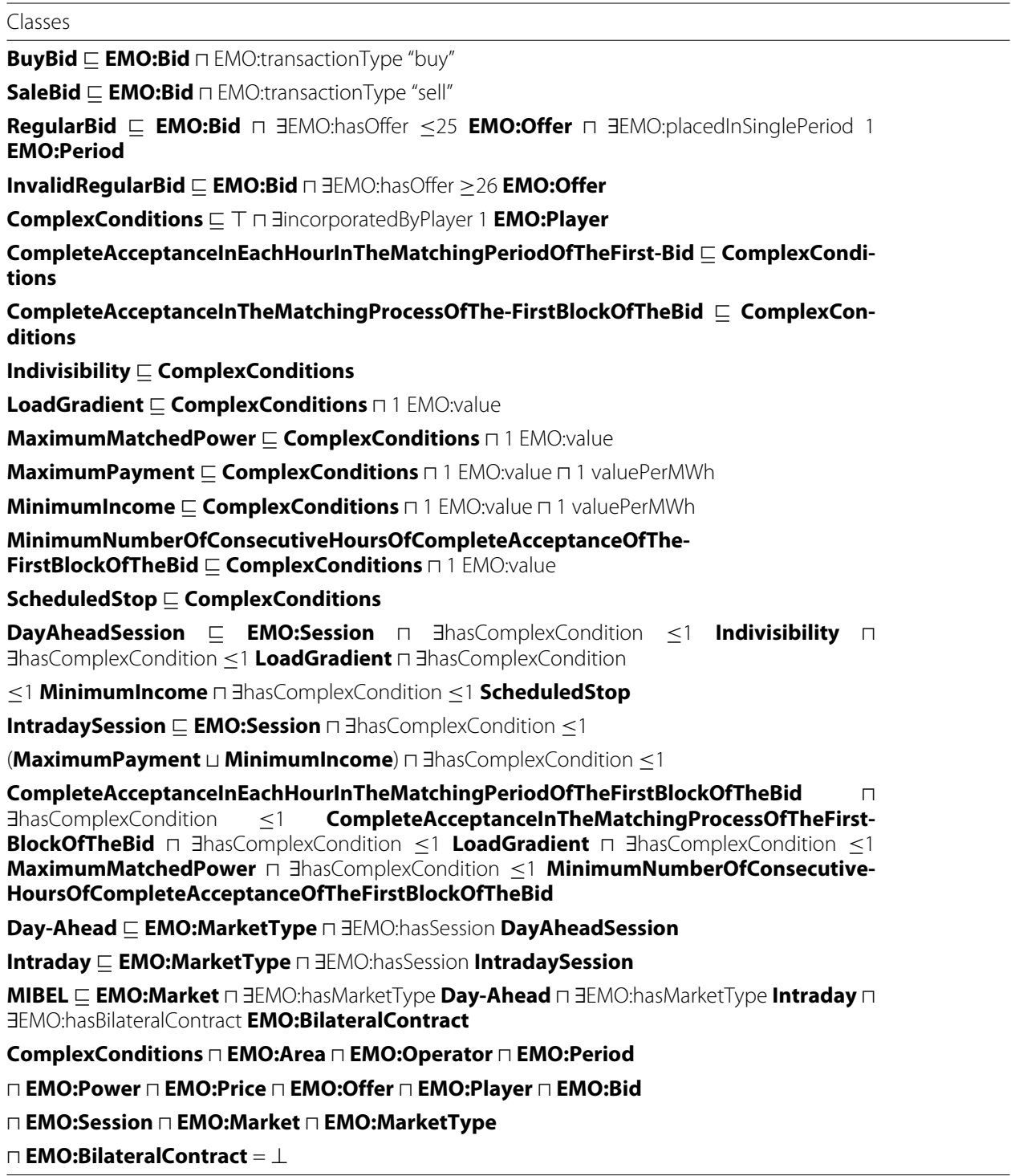


the object property EMO:hasOffer. It can only be related to an EMO:Period, making use of the EMO:placedInSinglePeriod Functional object property. On the other hand, an InvalidRegularBid in MLB ontology is defined as a EMO:Bid with more than 25 EMO:Offers.

The ComplexConditions concept includes the EMO:Player that places the constraints with the object property incorporatedByPlayer. The Indivisibility, the ScheduledStop, the CompleteAcceptanceInEachHourInTheMatchingPeriodOfTheFirstBlockOfTheBid and the CompleteAcceptanceInTheMatchingProcessOfTheFirstBlockOfTheBid are subclasses of ComplexConditions. The LoadGradient, the MaximumMatchedPower, and the MinimumNumberOfConsecutiveHoursOfCompleteAcceptanceOfT-heFirstBlockOfTheBid are also subclasses of ComplexConditions but include a EMO:value data property which can be a double value in the case of the first two conditions (representing the load amount) or an integer in the case of the last (representing the number of hours). The remaining complex conditions, i.e. MaximumPayment and MinimumIncome, in addition to being subclasses of ComplexCondition and including the EMO:value data property, are also defined by a valuePerMWh data property.

A DayAheadSession is subclass of EMO:Session and may include one or more of the complex conditions: Indivisibility, LoadGradient, MinimumIncome and ScheduledStop, using the object property hasComplexCondition. A IntradaySession is also subclass of EMO:Session and may include one or more of the following conditions: MaximumPayment or MinimumIncome (depending on being a buyer or seller agent respectively), LoadGradient, MaximumMatchedPower, CompleteAcceptanceInEachHourInTheMatchingPeriodOfTheFirstBlockOfTheBid, CompleteAcceptanceInTheMatchingProcessOfTheFirstBlockOfTheBid and MinimumNumberOfConsecutiveHoursOfCompleteAcceptanceOfTheFirstBlockOfTheBid.

The Day-Ahead and Intraday concepts are subclasses of EMO:MarketType, including the DayAheadSession and IntradaySession respectively, making use of the EMO:hasSession object property.

MIBEL is subclass of EMO:Market including the Day-Ahead and Intraday market types and also the EMO:BilateralContract, using EMO:hasMarketType and EMO:hasBilateralContract object properties respectively.

Finally, the ComplexConditions, the EMO:Area, the EMO:Operator, the EMO:Period, the EMO:Power, the EMO:Price, the EMO:Offer, the EMO:Player, the EMO:Bid, the EMO:Session, the EMO:Market, the EMO:MarketType and the EMO:BilateralContract classes are all Disjoint Classes, meaning that none of these classes has members in common.

\section{Case study}

The case study is focused on the Iberian EM, composed by Portugal and Spain. It includes offers from all players of the day-ahead market concerning the day of $1^{\text {st }}$ January, 2012. OMIE (Operador del Mercado Ibérico de Energía) (OMIE 2012; 2017), MIBEL's market operator, publishes the purchase and sale offers of each player after market clearance. The data regards 826 distinct players, from which 714 are sellers and the remaining 112 are buyers, and was gathered with the extraction tool proposed in Pereira et al. (2014). 
Special attention is paid to the agent SG 821, a MASGriP aggregator whose production is only based on wind generation. In this case study only communications concerning the interoperability between MASCEM and MASGriP are considered, since the remaining have already been presented in the previous works (Santos et al. 2016a, b, c).

After receiving the call for proposal sent by MASCEM's market operator for the dayahead market, player SG 821 gathers all the necessary information from its aggregated players, in order to submit a proposal to participate in the market.

From the point of view of the market operator, SG 821 is a common player of MASCEM, being the communications exchanged by these two agents similar to those exchanged with MASCEM's native players, i.e., using the same ontology.

Figure $4^{9}$ features the call for proposal sent by MASCEM's Market Operator to each registered player in $\mathrm{RDF}^{10}$.

Analysing Fig. 4 it is possible to see the definition of a call for proposal (lines 36-39) for the electricity market named MIBEL (lines 31-35), with market type SPOT (lines 11-17), operated by the OMIE market operator (lines 40-43), with a single day-ahead session

$<$ ?xml version="1.0" encoding="UTF -8 " standalone="no"?> $<$ rdf: RDF

xmlns: cfp="http://www. mascem. gecad.isep.ipp.pt/ontoloqies/call-for-proposal. owl\#"

xmlns : emo=" $h$ ttp://www. mascem. gecad.isep.ipp.pt/ontoloqies/electricity-markets. owl\#"

xmlns : mibel="http://www. mascem. gecad. isep. ipp.pt/ontoloqies/mibel.owl\#"

xmlns: owl="http: //www.w3.org/2002/07/owl\#"

xmlns : $r$ df="http://www.w3.orq/1999/02/22-rdf-syntax-ns\#"

xmlns: rdfs $=$ "http://www.w3.orq/2000/01/rdf-schema\#"

xmlns: xscl="http://www.w3. orq/2001/xML.Schema\#"

xml:base="http://www.mascem.gecad.isep.ipp.pt/ontologies/">

11 brdf:Description rdf: about="mibel.owl\#iMT-SPOT">

<emo:id rdf: datatype="http://www.w3.ord/2001/XMLSchema\#unsiqnedLong" >

$8779166223036938470</$ emo: id $>$

$<$ emo: name $>$ SPOT $</$ emo: name $>$

$<$ emo: hasSession rdf: resource="mibel. owl\# iDayAheadSession2012-01-01-0"/>

<emo:isoperatedBy raf:resource="electricity-markets.owl\#iMO-OMIE"/>

$\langle$ rdf: type rdf:resource="mibel.owl\#Day-Ahead"/>

$</$ rdf: Description $>$

<rdf:Description rdf : about="mibel.owl\#iDayAheadSession2012-01-01-0">

<emo: date rdf: datatype="http://www.w3.orq/2001/XMLSchema\#date" >2012-01-01 $</$ emo:date>

<emo:id rdf: datatype="http://www.w3.orq/2001/XML.Schema\#unsiqnedLong" ">

$2401209299201176490</$ emo: id $>$

<emo: number rdf : datatype="http://www .w3.ora/2001/XML.Schema\#uns iqnedInt" >0

$</$ emo: number $>$

<emo: numberofPeriods rdf : datatype="http://www.w3.org/2001/XMLSchema\#unsiqnedInt" >

$24</$ emo: numberofPeriods $>$

<emo:maxNumberofFractions rdf: datatype="

http://www. w3.orq/2001/XMLSchema\#unsi qnedInt" $>25</$ emo: maxNumberofFractions $>$ <rdf: type rdf: resource="mibel. owl\#DayAheadSession"/> $\langle/$ rdf : Description $\rangle$

<rdf:Description rdf: about="call-for-proposal.owl\#">

<rdf:type rdf:resource="http://www.w3.orq/2002/07/owl\#Ontology"/>

<owl: imports rdf:resource="call-for-proposal.owl\#"/>

<owl: imports raf: resource="mibel. owl\#"/>

$</$ rdf: Description $>$

$\langle$ rdf: Description rdf: about="mibel.owl\#iM-MIBEL">

<rdf:type rdf:resource="mibel.owl\#MIBEL"/>

$<$ <emo: name>MIBEL</emo: name>

<emo:hasMarketType rdf: resource="mibel. owl\#iMT - SPOT" / >

$</$ rdf : Description $>$

<rdf: Description rdf : about="call-for-proposal. owl\# iCFP-DayAheadSession2012-01-01-0" <rdf:type rdf:resource="call-for-proposal.owl\#CallForProposal"/>

<cfp:forElectricityMarket rdf: resource="mibel.owl\#iM-MIBEL"/> $</$ rdf: Description $>$

<rdf:Description rdf: about="electricity-markets.owl\#iMO-OMIE">

<rdf:type rdf:resource="electricity-markets. owl\#Marketoperator" / >

$<$ emo: name>OMIE</emo: name>

$</$ rdf: Description $>$

L/rdf:RDF

Fig. 4 OMIE Market Operator's call for proposal 
(lines 18-25) defining $24 \mathrm{~h}$ periods (line 22) and 25 as the maximum number of fractions (line 23) for the $1^{\text {st }}$ January, 2012 (line 19).

After gathering all the required information from it's aggregates, SG 821 generates its market proposal and sends it to OMIE market operator. Figure 5 presents a snippet of the proposal sent by SG 821 to the OMIE market operator. A full representation of the proposal is available online ${ }^{11}$.

Figure 5 shows the definition of a Bid placed in Period 1 (line 33), of transaction type sell (see line 30), for which 25 Offer fractions are defined (in lines 22-25, 27-29, 31, 32, and 34-49).

After receiving all the proposals or after ending the available time for the reception of bids, the market operator validates the proposals and executes the session of the daily market. At the end of the market simulation, it converts the market results' of each player into RDF and sends them to the respective player. A snippet of the market results of SG 821 is shown in Fig. 6. The full RDF is available online ${ }^{12}$.

By analysing the last lines of the RDF excerpt from Fig. 6, it is possible to see the definition of the traded power for period 11 with value 1302.2 and unit $M W$ (lines 29-33). The results of player SG 821 in the day-ahead market can also be visualized graphically in Fig. 7.

Analysing the results of SG 821 from Fig. 7, it is visible that all of the energy available for sale in the market has been negotiated. As the player's production is wind based, he has offered 0 price in each period, in order to dispatch all of the available generation. However, it is possible to observe that the market price of the first 5 periods is higher than expected. This is explained by the fact that, although player SG 821 had a significant amount of power to sell in the market, the remaining producers didn't, making the price to increase significantly.

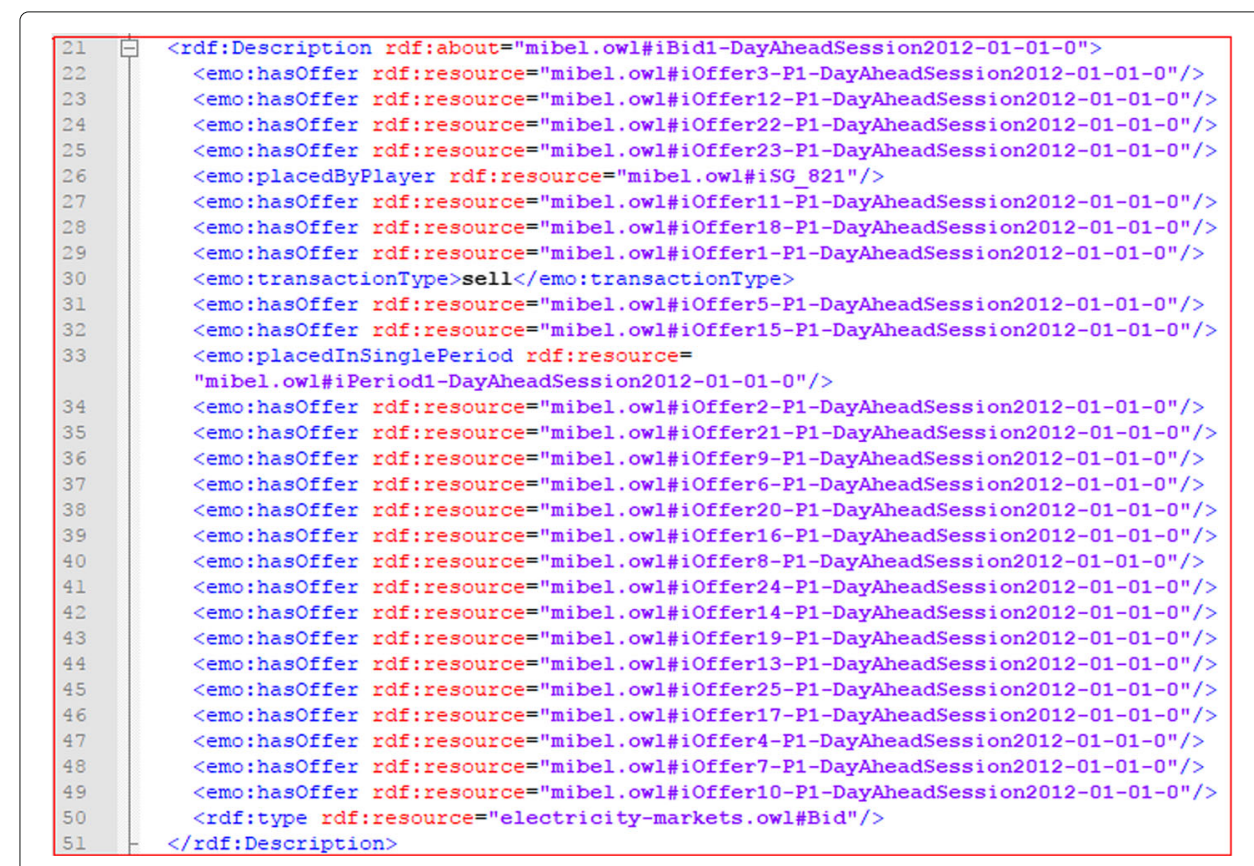

Fig. 5 Snippet of spot proposal presented by SG 821 


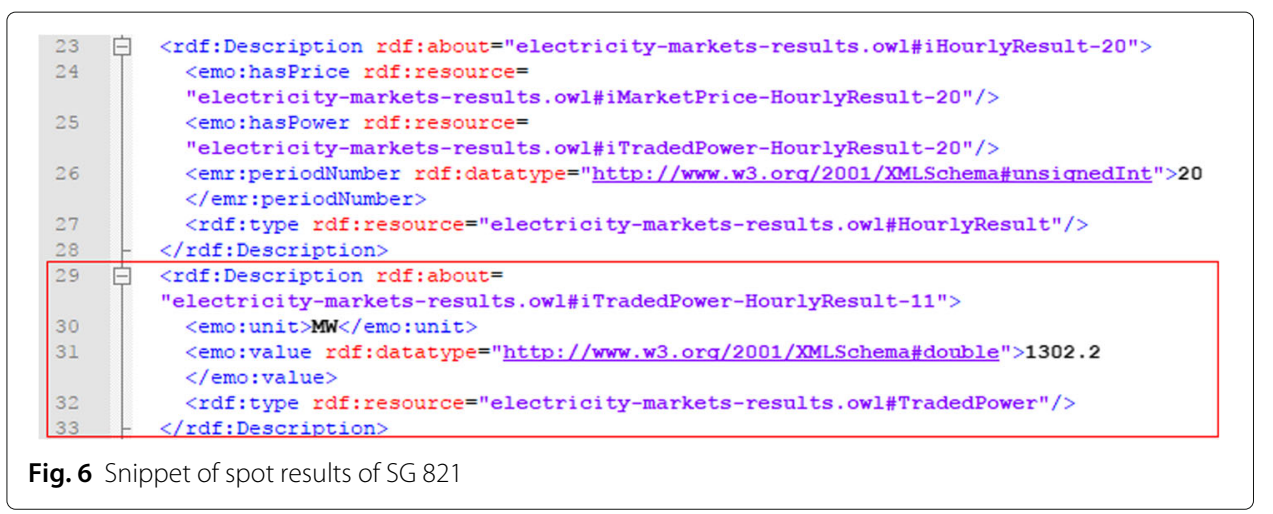

Making use of MASCEM's public ontology, MASGriP players have the opportunity to participate in the simulations of the wholesale EM of MASCEM. The publicly available ontology of MASCEM allows players from any MAS, or individuals, to participate in its simulations, like any player of the own MASCEM. Therefore, through MASCEM's ontology, the interoperability between MASCEM and agents from external systems willing to participate in the simulations of EM as market players is enabled.

\section{Conclusions}

MASCEM interacts with heterogeneous MAS developed within GECAD research group. Being these independent platforms, there is the need to interconnect them in order to enable the study of broader and complex scenarios. Additionally, opening the simulation environment to other systems brings the opportunity of integrating different market models and allows agents from external systems to interact in joint simulations. For such, it is mandatory that the messages exchanged by the involved agents may be properly interpreted by all. The cooperation between the different platforms can benefit in a large scale the realism and depth of EM and power systems' studies.

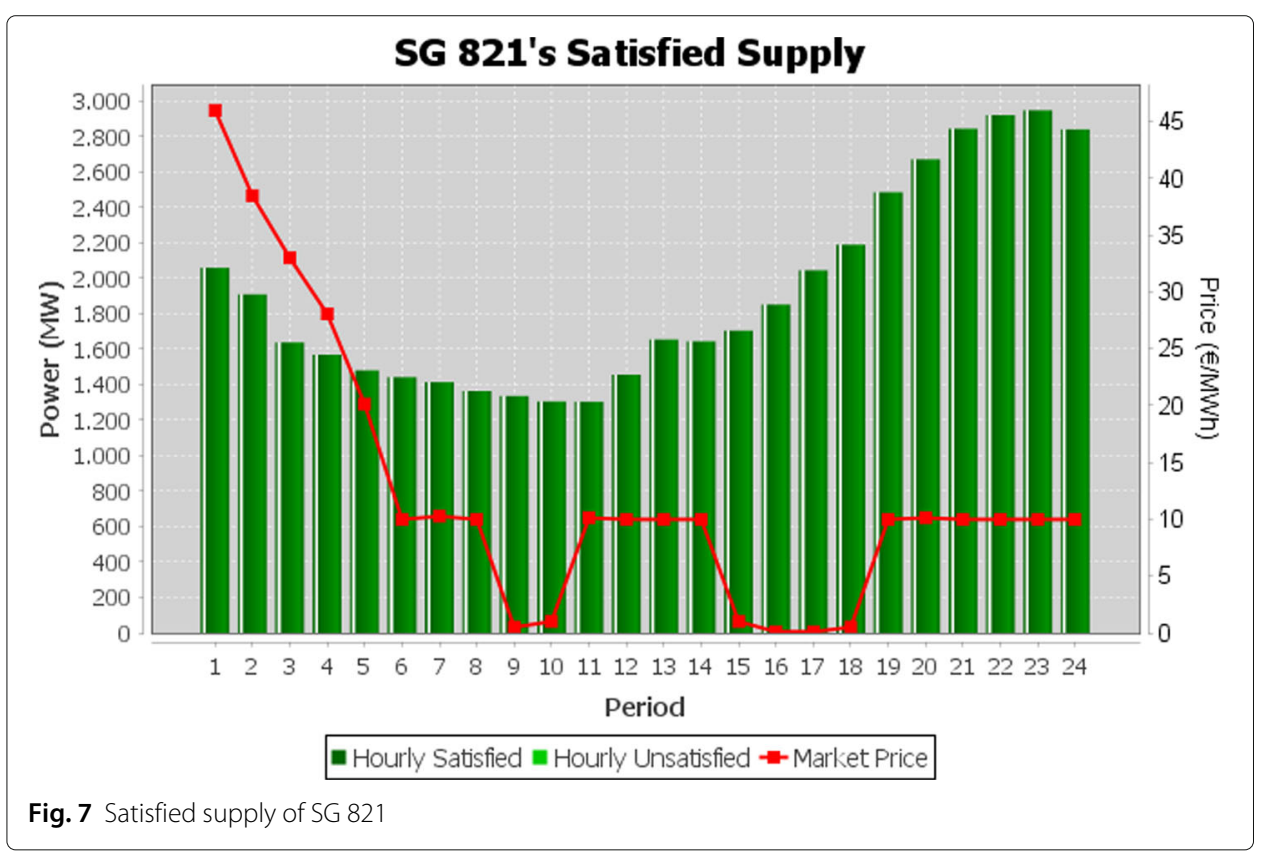


There are inherent difficulties in integrating independently developed agent-based systems, especially to access and map private ontologies. To overcome these difficulties, this work disseminates the development of interoperable MAS in the EM research area, thus enabling knowledge exchange between them in order to take full advantage of their functionalities, and promoting the adoption of a common semantic that enables the communication between these heterogeneous systems.

To this end, EMO has been developed. EMO is the base ontology from which several domain specific ontologies were extended. This is the case of the CFP, EMR and MBL. The first two are common ontologies for EM operation, while the last one is related to the MIBEL EM model included in MASCEM. The developed ontologies are publicly available to be easily accessed, reused and extended by Ontology Engineers or Agent-based systems developers in the scope of EM.

The presented case study demonstrates the interoperability between MASCEM and MASGriP, while illustrating the use and advantages of the developed ontology module. The emphasis in given to the communications exchanged between agents from MASCEM and MASGriP.

The integration of the proposed ontologies provides a solid and enhanced platform to study and explore the implications and consequences of new and already existing approaches in EM.

\section{Endnotes}

${ }^{1}$ http://www.mascem.gecad.isep.ipp.pt/ontologies

${ }^{2}$ http://www.mibel.com/

${ }^{3}$ https://www.epexspot.com/

${ }^{4}$ http://www.nordpoolspot.com/

${ }^{5}$ http://www.mascem.gecad.isep.ipp.pt/ontologies/mibel.owl

${ }^{6} \mathrm{http}$ //www.mascem.gecad.isep.ipp.pt/ontologies/paper/energyinf/18/mbl.png

${ }^{7}$ http://www.obitko.com/tutorials/ontologies-semantic-web/owl-dl-semantics.html

${ }^{8} \mathrm{~A}$ functional property is a property that only relates the same subject to one single object/value.

${ }^{9}$ http://www.mascem.gecad.isep.ipp.pt/ontologies/paper/energyinf/18/cfp.rdf

${ }^{10}$ https://www.w3.org/RDF/

${ }^{11}$ http://www.mascem.gecad.isep.ipp.pt/ontologies/paper/energyinf/18/proposal.rdf

${ }^{12}$ http://www.mascem.gecad.isep.ipp.pt/ontologies/paper/energyinf/18/result.rdf

Funding

This work has received funding from the European Union's Horizon 2020 research and innovation programme under the Marie Sklodowska-Curie grant agreement No 641794 (project DREAM-GO) and from FEDER Funds through COMPETE program and from National Funds through FCT under the project UID/EEA/-00760/2013. SFRH/BD/1 18487/2016 (Gabriel Santos PhD).

Availability of data and materials

The datasets generated during and/or analysed during the current study are available from the corresponding author on reasonable request. A significant part of these data is publicly available at www.mibel.com and (Santos 2015). The ontologies presented in this work are publicly available at www.mascem.gecad.isep.ipp.pt/ontologies.

Authors' contributions

GS conceived and developed the proposed ontologies, implemented them in the multi-agent systems, conducted the experiments and wrote the paper. TP contributed in the conception of the ontologies, design of the interactions between the systems, design of the experiments, analysis of results and writing of the manuscript. IP contributed in the overall design of the system, design of the experiments, use of data, draft and review of manuscript. ZV helped conceiving of the study, and participated in its design and coordination and helped to draft the manuscript. All authors read and approved the final manuscript. 


\section{Competing interests}

The authors declare that they have no competing interests.

\section{Publisher's Note}

Springer Nature remains neutral with regard to jurisdictional claims in published maps and institutional affiliations.

\section{Author details}

${ }^{1}$ GECAD - Research Group on Intelligent Engineering and Computing for Advanced Innovation and Development, Institute of Engineering - Polytechnic of Porto (ISEP/IPP), Rua Dr. António Bernardino de Almeida, 431, 4200-072 Porto, Portugal. ${ }^{2}$ BISITE research group - University of Salamanca, Calle Espejo, s/n, 37007 Salamanca, España. ${ }^{3}$ Polytechnic of Porto, Porto, Portugal.

Received: 12 February 2018 Accepted: 7 August 2018

Published online: 06 September 2018

\section{References}

Alexopoulos P, Kafentzis K, Zoumas C (2009) Elmo: An interoperability ontology for the electricity market. In: Proceedings of the International Conference on e-Business Milan, Italy. pp 15-20

Baader F, Horrocks I, Sattler U (2008) Chapter 3 description logics. In: Handbook of Knowledge Representation. Elsevier. pp 135-179

Bennaceur A, Andriescu E, Cardoso RS, Issarny V (2015) A unifying perspective on protocol mediation: interoperability in the future internet. J Internet Serv Appl 6(1):12

Biggar D, Hesamzadeh M (2014) The Economics of Electricity Markets. Wiley - IEEE. Wiley

Dong T, Duc CL, Lamolle M (2017) Tableau-based revision for expressive description logics with individuals. Web Semant Sci Serv Agents World Wide Web 45:63-79

Gomes L, Faria P, Morais H, Vale Z, Ramos C (2014) Distributed, agent-based intelligent system for demand response program simulation in smart grids. IEEE Intell Syst 29(1):56-65

Hippolyte JL, Howell S, Yuce B, Mourshed M, Sleiman HA, Vinyals M, Vanhee L (2016) Ontology-based demand-side flexibility management in smart grids using a multi-agent system. In: 2016 IEEE International Smart Cities Conference (ISC2). pp 1-7

Hopkinson K, Wang X, Giovanini R, Thorp J, Birman K, Coury D (2006) EPOCHS: A platform for agent-based electric power and communication simulation built from commercial off-the-shelf components. IEEE Trans Power Syst 21 (2):548-558

Kakran S, Chanana S (2018) Smart operations of smart grids integrated with distributed generation: A review. Renewable and Sustainable Energy. Reviews 81:524-535

Lin H, Veda SS, Shukla SS, Mili L, Thorp J (2012) GECO: Global event-driven co-simulation framework for interconnected power system and communication network. IEEE Transactions on Smart Grid 3(3):1444-1456

Maffei A, Srinivasan S, Castillejo P, Martínez JF, lannelli L, Bjerkan E, Glielmo L (2017) A semantic-middleware-supported receding horizon optimal power flow in energy grids. IEEE Trans Ind Inform 14:35-46

Man D (2013) Ontologies in computer science. Didactica Math 31:43-46

Meeus L, Purchala K, Belmans R (2005) Development of the internal electricity market in europe. Electr J 18(6):25-35

Oliveira P, Pinto T, Morais H, Vale Z (2012) Masgrip - a multi-agent smart grid simulation platform. In: 2012 IEEE Power and Energy Society General Meeting. pp 1-8

OMIE (2017) Omie, markets and products, electricity market, about our market. http://www.omie.es/en/home/marketsand-products/about-our-market. Accessed 18 May 2017

OMIE (2012) Omie report, daily and intraday electricity market operating rules. http://www.omel.es/files/ reglas_agosto_2012_ingles.pdf. Accessed 19 May 2017

Pereira IF, Sousa TM, Praça I, Freitas A, Pinto T, Vale Z, Morais H (2014) Data Extraction Tool to Analyse, Transform and Store Real Data from Electricity Markets. Springer International Publishing, Cham

Praça I, Ramos C, Vale Z, Cordeiro M (2003) Mascem: a multiagent system that simulates competitive electricity markets. IEEE Intell Syst 18(6):54-60

Ritschel B, Borchert F, Kneitschel G, Neher G, Schildbach S, lyemori T, Koyama Y, Yatagai A, Hori T, Hapgood M, Belehaki A, Galkin I, King T (2016) Experiments using semantic web technologies to connect iugonet, espas and gfz isdc data portals. Earth, Planets and Space 68(1):181

Santos G (2015) Ontologies for the interoperability of multiagent electricity markets simulation platforms. Master's thesis, Instituto Superior de Engenharia do Porto, Porto 11. http://hdl.handle.net/10400.22/8200

Santos G, Pinto T, Morais H, Praça I, Vale Z (2011) Complex market integration in mascem electricity market simulator. In: 2011 8th International Conference on the European Energy Market (EEM). pp 256-261

Santos G, Pinto T, Praça I, Vale Z (2016a) Mascem: Optimizing the performance of a multi-agent system. Energy 111:513-524

Santos G, Pinto T, Praça I, Vale Z (2016b) An interoperable approach for energy systems simulation: Electricity market participation ontologies. Energies 9(11):878

Santos G, Pinto T, Vale Z, Morais H, Praça I (2012) Balancing market integration in mascem electricity market simulator. In: 2012 IEEE Power and Energy Society General Meeting. pp 1-8

Santos G, Pinto T, Vale Z, Praça I, Morais H (2016a) Electricity Markets Ontology to Support MASCEM's Simulations. Springer International Publishing, Cham. pp 393-404

Santos G, Pinto T, Vale Z, Praça I, Morais H (2016c) Enabling communications in heterogeneous multi-agent systems: Electricity markets ontology. ADCAIJ: Advances in Distributed Computing and Artificial Intelligence Journal 5(2)

Schachinger D, Kastner W, Gaida S (2016) Ontology-based abstraction layer for smart grid interaction in building energy management systems. In: 2016 IEEE International Energy Conference (ENERGYCON). pp 1-6 
Scherfke S (2018) Mosaik documentation. https://media.readthedocs.org/pdf/mosaik/latest/mosaik.pdf. Accessed Jan 2018

Schütte S, Scherfke S, Tröschel M (2011) Mosaik: A framework for modular simulation of active components in smart grids. In: 2011 IEEE First International Workshop on Smart Grid Modeling and Simulation (SGMS). pp 55-60

Sioshansi F (2013) Evolution of Global Electricity Markets: New paradigms, new challenges, new approaches. Elsevier Sci Vale Z, Pinto T, Morais H, Praça I, Faria P (2011) Vpp's multi-level negotiation in smart grids and competitive electricity markets. In: 2011 IEEE Power and Energy Society General Meeting. pp 1-8

Veeckman C, Jedlička K, De Paepe D, Kozhukh D, Kafka Š, Colpaert P, Cerba O (2017) Geodata interoperability and harmonization in transport: a case study of open transport net. Open Geospatial Data Softw Stand 2(1):3

Submit your manuscript to a SpringerOpen ${ }^{\odot}$ journal and benefit from:

- Convenient online submission

$\rightarrow$ Rigorous peer review

- Immediate publication on acceptance

- Open access: articles freely available online

- High visibility within the field

- Retaining the copyright to your article

Submit your next manuscript at $>$ springeropen.com 